\title{
Changements organisationnels et communication dans une institution financière coopérative $d u$ Québec
}

\section{Guy Cameron}

\section{(2) OpenEdition}

Journals

Édition électronique

URL : http://journals.openedition.org/communicationorganisation/1637

DOI : 10.4000/communicationorganisation. 1637

ISSN : $1775-3546$

Éditeur

Presses universitaires de Bordeaux

Édition imprimée

Date de publication : 1 mai 1993

ISSN : 1168-5549

\section{Référence électronique}

Guy Cameron, "Changements organisationnels et communication dans une institution financière coopérative du Québec », Communication et organisation [En ligne], 3 | 1993, mis en ligne le 26 mars 2012, consulté le 19 avril 2019. URL : http://journals.openedition.org/

communicationorganisation/1637 ; DOI : 10.4000/communicationorganisation.1637

Ce document a été généré automatiquement le 19 avril 2019

(c) Presses universitaires de Bordeaux 


\title{
Changements organisationnels et communication dans une institution financière coopérative du Québec
}

\author{
Guy Cameron
}

1 Alors que le vingtième siècle mettait la surface du globe à feu et à sang, qu'il déplaçait sans ménagement les frontières de plusieurs États, qu'il découvrait dans l'infiniment petit une extraordinaire source d'énergie, mais aussi un pouvoir meurtrier terrifiant, qu'il révolutionnait les mœurs et qu'il ravalait les fictions les plus audacieuses au rang d'inoffensives histoires pour enfants, le monde bancaire, lui, semblait figé dans ses allures dix-neuvième.

2 Il y eut bien, de temps à autre, quelques soubresauts, lors de la Grande Dépression, par exemple; mais ces crises ne duraient, aurait-on dit, que le temps nécessaire pour punir ceux qui avaient transgressé la discipline traditionnelle du milieu et mettre les autres en garde contre toute tentation de frivolité financière.

3 D'une génération à l'autre, la banque gardait son protocole empesé, ses boiseries, ses colonnades altières, satisfaite de voir se lisser lentement devant ses comptoirs patinés la pierre de ses parquets. Autour d'elle, de petites institutions nouvelles, caisses d'épargne, coopératives de crédit et diverses sociétés financières, ramassaient les miettes, rêvant du jour où elles pourraient, à leur tour, contempler leurs armoiries avec autant de contentement et de sérénité. Mais...

4 Mais survint, dans les années 1950, une frénésie de consommation sans précédent, qui transforma rapidement la situation et les habitudes financières des ménages et des entreprises. À travers cet élan de croissance, prometteur d'un avenir fascinant ouvert par la technique, s'amorça bientôt l'invasion de l'informatique, qui allait de façon inattendue modifier une grande partie des pratiques de la banque. Et les changements ne faisaient que commencer. 
5 Peu de temps après, dans les années 1970, ce furent les bases mêmes de la géo finance qui furent ébranlées par les ondes du choc pétrolier: nombre de lois apparemment immuables, nombre de certitudes qu'on se transmettait avec assurance se révélaient soudain fragiles, sinon nettement dépassées. Entre-temps, les télécommunications avaient déjà fait alliance avec les ordinateurs pour obliger les institutions financières à forcer la cadence de leurs modes habituels de transfert de fonds et de garanties. Du coup se trouvaient anachroniques de nombreux circuits établis depuis des décennies et tronquées de vénérables pièces sur lesquelles reposait depuis longtemps la quiétude des opérations de vérification et de contre-vérification si chères au monde bancaire; du coup, également, devenait désuète et encombrante une bonne partie de la volumineuse paperasse dont on s'était fait avec le temps, dans les banques, un cocon blindé.

6 Naturellement, le jeu de la concurrence et les pressions de la rentabilité aidant, l'automatisation de certaines catégories d'opérations et le libre-service ne tardèrent pas à apparaître, annonçant une transformation profonde des relations habituelles de la banque avec ses clients. En même temps, à la faveur des technologies nouvelles, surgirent aussi les réseaux interinstitutionnels, puis les réseaux internationaux dont l'évolution permit, en quelques années, de dégeler les capitaux et de les faire voyager plus facilement encore que les personnes. En quelques secondes maintenant, des masses de capital considérables peuvent être déplacées d'un continent à l'autre et, peu importe l'endroit où l'on se trouve, il est désormais possible d'obtenir presque instantanément des renseignements précis sur la crédibilité financière d'une personne ou d'une organisation... À tout cela allaient encore s'ajouter des variables nouvelles suscitées par l'internationalisation des activités de plusieurs grandes institutions, l'endettement des particuliers et des États, l'incapacité de plusieurs pays en développement de rembourser leurs dettes, la mise en marché d'un grand nombre de nouveaux services et de nouvelles formes de financement, le décloisonnement graduel des champs d'activités financières, un resserrement marqué des marges de bénéfice, la révision des législations nationales et l'instauration de plusieurs réglementations internationales.

7 Si le milieu des services financiers vit, depuis vingt ou trente ans, une telle diversité de changements, ce n'est pas forcément qu'il soit en train de s'installer déjà dans le vingt-etunième siècle. C'est plutôt qu'il a mis bien du temps à entrer dans le vingtième. Mais depuis qu'il s'y est engagé, la cadence des changements lui a laissé bien peu de répit.

L'objet de cet article, c'est d'illustrer certains des rôles que la communication organisationnelle a pu jouer dans l'adaptation d'une institution financière à ces multiples exigences de l'évolution de son secteur d'activité.

\section{Le cas du Mouvement des caisses Desjardins}

9 Le milieu d'observation sera, plus précisément, le réseau coopératif québécois du Mouvement des caisses Desjardins. Cette institution est formée, à la base, de 1475 coopératives de services financiers, appelées caisses populaires (caisses locales) ou caisses d'économie (caisses de groupes) Desjardins, réunies au sein de quatorze fédérations (une fédération de caisses d'économie et treize fédérations régionales de caisses populaires), elles-mêmes rassemblées par une confédération qui chapeaute également une vingtaine de sociétés de services complémentaires. Ce groupe financier, administré par 18000 dirigeants bénévoles élus par les membres des caisses, gère un actif global de soixante 
milliards de dollars canadiens (soit environ 250 milliards de francs français). Ses activités quotidiennes requièrent le concours de 40000 employés, appelés à servir cinq millions de membres-sociétaires qui sont à la fois les usagers et les propriétaires des caisses et de leurs filiales. Il est important de signaler que ce réseau, en vertu de sa nature coopérative, est administré suivant des règles démocratiques qui commandent un degré spécial de participation et de décentralisation, donc d'information et de communication.

En plus de tous les changements qui ont touché l'ensemble des grandes institutions financières contemporaines, le Mouvement des caisses Desjardins a connu de nombreuses modifications internes découlant de sa dynamique propre. Par exemple, depuis trente ans, sa loi constitutive a été modifiée à plusieurs reprises (notamment en 1968, 1970, 1978 et 1981) et entièrement refondue deux fois, soit en 1963 et en 1988. Toutes les dispositions réglementaires ont dû, évidemment, être chaque fois révisées. Depuis 1964, l'actif global a été multiplié par cinquante et l'organigramme du Mouvement s'est enrichi d'une quinzaine de nouvelles filiales. Le système comptable en usage dans les caisses a été complètement remodelé à trois reprises. Environ cent cinquante nouveaux services ont été créés, plusieurs générations d'équipements informatiques et de logiciels se sont succédées et toutes les normes et méthodes de capitalisation ont été revues. Tout cela se déroulait alors que le Mouvement enregistrait une croissance annuelle moyenne d'environ $15 \%$, qui sollicitait constamment des ajouts de personnel et d'équipements, de nouvelles répartitions de tâches, des réorganisations internes, des réaménagements physiques et de nouveaux systèmes de gestion et de contrôle.

\section{Changement et contexte}

Globalement, on peut dire que, dans le Mouvement des caisses Desjardins, ces changements ont été vécus la plupart du temps de manière harmonieuse, à l'occasion même avec enthousiasme. Il y a à cela plusieurs explications.

La première, c'est que l'institution était en pleine croissance. Dans tous les replis de ses structures, on pouvait avoir l'impression de bâtir, de progresser, d'élargir l'influence et l'action de l'organisation, de gagner du terrain sur la concurrence, d'établir de nouveaux standards de performance. À partir d'un ensemble de petites unités pour la plupart récentes, modestes, artisanales même, on était en train de monter un réseau d'envergure, dont l'image et la réputation se raffermissaient sans cesse, au rythme de l'accroissement de ses capacités financières et de son capital de compétence.

L'un des effets de cette croissance était qu'elle permettait d'absorber, moyennant un minimum de mesures de perfectionnement, les surplus de personnel qu'aurait pu entraîner l'adoption des nouvelles technologies et façons de faire. On comprend dès lors que, de manière générale, le changement technologique n'a pas vraiment eu l'occasion, dans ce réseau, de s'alourdir des connotations menaçantes qu'il s'est si souvent accolées dans nombre d'autres secteurs de l'économie.

Il y a aussi que cette modernisation et cette expansion de l'institution étaient considérées, aussi bien en dehors qu'en dedans, comme un élément clé du projet d'affirmation nationale du Québec. Dans différents milieux (formations politiques, syndicats, média, universités...), on citait de plus en plus fréquemment le Mouvement comme exemple de dynamisme, d'audace, d'innovation, comme un des prototypes du modèle de développement des Québécois et comme l'un des principaux lieux de concertation et de 
ralliement des éléments les plus entreprenants de la collectivité. Au fur et à mesure que se renforçaient son savoir-faire ainsi que ses moyens techniques et financiers, le Mouvement prenait donc figure de levier économique et financier majeur pour la société québécoise et de chantier d'avenir stratégique pour chacune de ses communautés locales ou régionales.

Ce caractère de « réalisation collective » du Mouvement des caisses Desjardins suscitait une fierté croissante parmi ses membres-usagers et dans la population en général, donc à plus forte raison aussi parmi ses dirigeants et ses employés. Régulièrement, les hauts dirigeants du Mouvement attiraient l'attention de ces derniers sur la notoriété nouvelle de LEUR organisation, invitant chacun d'eux à y reconnaître sa part de mérite, de succès et de prestige. On devine facilement les effets mobilisants que ces renforcements pouvaient avoir sur le moral des troupes et l'ardeur de chacun dans la réalisation des changements essentiels à l'évolution de l'organisation.

Dans ce sillage, plusieurs des principaux dirigeants du Mouvement furent tour à tour des figures publiques dominantes, écoutées et respectées. Ces leaders ont souvent été, en fait, au sein du Mouvement comme dans l'ensemble de la société québécoise, d'influents promoteurs de changement, tant sur le plan de la vision que cette société avait d'ellemême que dans le domaine plus étroit de l'économie et de la finance. Ils formulaient des objectifs communs audacieux et stimulants devant lesquels, graduellement, l'évolution même du Mouvement prenait figure de symbole : celui de la force de la solidarité et des espoirs permis lorsqu'on regarde l'avenir avec confiance, celui de la capacité même de la collectivité québécoise à maitriser davantage son économie et sa vie politique.

Pas toujours dénué des traits un peu outranciers du prophétisme et du triomphalisme, le discours externe et interne des dirigeants du Mouvement avait cependant, malgré ses enflures et d'occasionnelles incohérences, l'effet de stimuler le travail des dirigeants bénévoles et des employés. Au cœur des tâches les plus terre à terre du quotidien, il plaçait des préoccupations nobles et des idéaux élevés devant lesquels tous, du premier leader au plus humble des employés, se voyaient conférer des responsabilités. Il entretenait le goût de foncer, d'imaginer des moyens nouveaux, des façons de faire nouvelles, de garder la tête du peloton, sur le plan technologique, de conquérir de nouveaux champs d'action, d'acquérir de nouvelles compétences, de répondre avec empressement aux attentes des membres et de la collectivité.

On perçoit là le rôle complexe et " pluriel » d'une institution coopérative influente, dans lequel s'entremêlent constamment, à des doses fluctuantes, les ambitions d'un réseau d'entreprises en développement et les idéaux stimulants d'un mouvement de transformation économique et sociale. Seules, sans doute, pouvaient alors prétendre à un tel rôle dans la collectivité québécoise des institutions ou entreprises de nature collective comme les grandes coopératives ou quelques sociétés d'État de création récente; largement porté par le courant nationaliste, le Mouvement y trouva pendant ces années dynamisme, inspiration et complicités. Il se chargea, en même temps, de multiples attentes qui, avec le temps, lui tailleraient une mission large et généreuse, mais exigeante et jusqu'à un certain point périlleuse. 


\section{Importance de la consultation}

19 Le cheminement du Mouvement des caisses Desjardins avait au moins un autre trait particulier, celui d'être en grande partie inspiré par la base même de l'organisation, celle des membres, des dirigeants qui les représentent et des employés engagés à leur service. C'est une chose que de réaliser des changements du haut en bas d'une organisation pyramidale, c'en est une autre que de rendre possible la réalisation de changements demandés ou souhaités par les personnes mêmes qui seront touchées par les changements ou appelées à les concrétiser. Dans le Mouvement des caisses Desjardins, ce sont les gens de caisses, dirigeants et employés, qui sont sur la ligne de front, en contact permanent avec les usagers. C'est souvent de cette source que sont venues les volontés de changement, exprimées tantôt par les employés, tantôt par les dirigeants, tantôt par les deux groupes, souvent réunis pour les besoins de l'action. Cette concertation connaissait régulièrement des temps forts au moment des grands congrès triennaux rassemblant des centaines, parfois même des milliers de personnes de tous les paliers de l'organisation.

Ces congrès du Mouvement ont souvent été les ressorts déterminants des grands changements qui ont marqué ses orientations, ses activités ou ses méthodes. Ils n'ont pas toujours dégagé des pistes claires, mais ils ont périodiquement permis à tous les groupes de personnes engagées dans le réseau de comparer leurs perceptions et opinions et de s'initier par étapes à de nouvelles façons de voir et de comprendre. L'harmonisation des attentes n'était pas toujours facile, les caisses étant implantées dans des milieux fort divers, dont les besoins étaient multiples et disparates. Ces attentes ne coïncidaient pas toujours, non plus, avec les évaluations faites par les experts des organismes de regroupement. De façon générale, toutefois, le Mouvement a su tirer parti de cette diversité des points de vue et surmonter les difficultés avec efficacité, multipliant les consultations et les opérations d'information, soumettant aussi ses grandes décisions à une certaine pratique du consensus. Souvent, les décisions ont tardé, ralenties par la nécessité de reprendre des cheminements, de refaire des preuves, de recommencer les pourparlers ou les études, parfois même par l'obligation d'attendre que s'amenuisent des oppositions ou des résistances. Par la suite, cependant, ces retards ont dans plusieurs cas été comblés par une efficacité foudroyante de l'action, grâce, sans doute, à l'abondance de l'information diffusée au cours du processus et au sentiment partagé que chacun avait pu, à un moment ou l'autre, exprimer son opinion, ses réserves, ses attentes et ses préoccupations. Il semble bien que, dans la gestion du changement, le temps qu'on investit à le préparer réduit au moins d'autant celui qu'on doit mettre à le réaliser

\section{La communication interne}

21 Le fonctionnement démocratique d'un réseau comme celui du Mouvement des caisses Desjardins ne peut reposer que sur une grande diversité de mécanismes de communication interne, tantôt formels, tantôt laissés au gré des circonstances et des faisceaux de relations fonctionnelles et interinstitutionnelles. Plusieurs caisses ont leur propre bulletin de communication avec leurs membres. Chacune a ses mécanismes d'information auprès de ses dirigeants (de onze à vingt) et de ses employés (de deux à deux cents). Chaque fédération de caisses dispose à son tour de périodiques d'information générale et de toute une variété de publications dont la facture et la fréquence sont 
réglées selon les besoins précis qui les ont fait naître. La Confédération et les sociétés complémentaires émettent, elles aussi, une grande quantité de publications et de documents divers. Occasionnellement rationalisées pour éliminer des chevauchements et des coûts inutiles, ces publications ont constamment tendance à se développer et à se remultiplier pour s'adapter aux besoins spécifiques de sous-publics ou à ceux de nouveaux champs d'action.

Depuis une douzaine d'années, la plupart des changements majeurs touchant l'ensemble du réseau ont fait l'objet de plans globaux de communication. Ce fut le cas, notamment, lors de l'acquisition de la franchise VISA, de la réalisation du programme de refonte du système informatique central et de l'implantation des changements découlant de la nouvelle loi de 1988. Ces opérations sont en général soutenues par des publications spéciales, à durée limitée, qui attirent l'attention des publics internes sur des aspects spécifiques des changements à mettre en œuvre. À ce mécanisme se greffent au besoin des sessions de formation ou d'information qui descendent en cascades, du lieu d'origine des modifications jusqu'aux caisses, où, généralement, doivent s'opérer les changements prévus.

Évidemment, dans les campagnes majeures, mais également dans le quotidien des opérations, tous ces canaux d'information et de formation se rejoignent, s'entrecoupent, se complètent, se répètent même. Ces répétitions ne sont pas toujours prévues, ni toujours voulues en raison des coûts qu'elles entraînent; mais, l'expérience l'a maintes fois démontré, la répétition est au cœur de la pédagogie, et la décentralisation de l'action, par le foisonnement des initiatives auquel elle donne souvent lieu, a fréquemment des effets bénéfiques inattendus. La diversité des sources et des modes de traitement de l'information finit aussi, la plupart du temps, par mettre en lumière les aspects essentiels des sujets traités, les dégageant graduellement des détails dont on les avait parfois surchargés au départ.

On affirme souvent que, dans le domaine de l'information, trop, c'est comme pas assez. C'est sans doute exact, à certains égards, notamment en ce qui a trait à la quantité et à la qualité des éléments cognitifs retenus. Mais il en est autrement sur le plan des attitudes. Si on développe facilement de l'agressivité, de l'hostilité, de la méfiance envers ceux qui semblent retenir ou dissimuler l'information, il est bien difficile d'en vouloir à ceux qui en diffusent trop. Les deux situations sont donc loin de créer les mêmes réactions émotives quand vient le temps de s'engager sur le plan de l'action.

Dans le Mouvement des caisses Desjardins comme dans la majorité des autres organisations ou entreprises, la préoccupation pédagogique n'est pas toujours manifeste dans la mise en œuvre de tous les changements.

L'ampleur des tâches commande sans arrêt ; l'urgence et la diversité des sujets à traiter précipitent souvent l'action. Mais la structure «politique » d'un réseau comme celui du Mouvement Desjardins a aussi ses lois. Pour protéger leur crédibilité et leur leadership, les principaux dirigeants de chaque niveau ont besoin d'ajuster constamment leurs orientations et leurs décisions aux attentes et aux opinions de leurs commettants. D'une part, ils n'ont pas la possibilité d'ignorer longtemps les désirs de ceux qui les élisent ou les nomment à leur poste ; d'autre part, s'ils veulent, dans l'intérêt de l'organisation, devancer l'expression des besoins de la base, il leur faut établir leurs orientations sur des études et réflexions approfondies et préparer les décisions par un patient travail d'information et de persuasion. Durant ce temps, les hypothèses se raffinent, les 
perspectives se corrigent, alors que l'information se diffuse, progressivement, dans les divers circuits de l'organisation. D'une certaine manière, la fragilité des pouvoirs délégués force les dirigeants et les gestionnaires à une véritable pédagogie de l'action qui, malgré les délais qu'elle implique, ne peut que favoriser en même temps l'efficacité pratique de l'implantation des changements.

\section{Une expérience déterminante du changement}

Si, dans le rayon de la gestion du changement, le Mouvement des caisses Desjardins a à son crédit des réussites certaines et un parcours qui fait parfois envie, c'est dû, en bonne partie, à sa nature d'organisation coopérative, démocratique et décentralisée, ainsi qu'à l'obligation que lui fait cette particularité constitutive de porter attention aux personnes. Cela ne signifie pas pour autant que tous les changements dont il a été l'objet aient été également réussis ni qu'il soit à jamais protégé de l'erreur et de l'échec.

Il faut cependant le mentionner, l'institution a globalement tiré des leçons éclairantes et précieuses de l'une des premières grandes transformations auxquelles elle a été contrainte, soit celle de l'informatisation de sa gestion de données. En 1965, l'accroissement du volume des opérations et la complexification de la gestion de l'information l'obligeaient à mettre sur pied un comité multipartite ayant pour mandat de proposer les meilleurs moyens de mécaniser les opérations comptables des caisses. Les travaux du comité le conduisirent à recommander l'informatisation du traitement de l'ensemble des données comptables. Entre-temps, les organisateurs de l'exposition universelle de Montréal ("Terre des Hommes»), qui devait se tenir en 1967, étaient à la recherche d'une institution financière qui accepterait le défi d'implanter sur le site de l'exposition une succursale de services bancaires à la fine pointe du développement. Le Mouvement risqua l'aventure. Ce fut, au Canada, la première expérience de télétraitement informatique des données dans le domaine financier. Bien que l'expérience fût difficile et risquée, ses résultats confirmèrent le bien-fondé des recommandations du comité sur la mécanisation des opérations. Il ne restait plus qu'à convaincre le réseau de la faisabilité et de la pertinence du projet.

On y mit pas moins de deux ans de réunions d'information et de consultations diverses, des centaines de pages de documents, de nombreux outils audio-visuels et plusieurs tournées de sensibilisation à travers tout le réseau. On n'amorça l'implantation de l'informatique qu'à partir du moment où la majorité accepta qu'on entame l'expérience, aucune caisse n'étant d'avance obligée de s'engager à accepter l'informatisation. Ce sont les résultats, à la fois fascinants et supérieurs aux plus optimistes prévisions, qui firent le reste. Mais les résistances ne tombèrent pas du jour au lendemain. Il fallut près d'une douzaine d'années pour informatiser tout le réseau, les questions de coûts étant aussi à considérer dans le cas des plus petites unités ; le partage équitable des coûts, sur une base de péréquation, permit finalement l'informatisation complète et uniforme de toutes les caisses, qui s'acquirent dès lors l'image d'un réseau unifié et d'avant-garde.

Parmi les réserves suscitées par un tel projet, il y avait, bien sûr, la crainte qu'il s'agisse là, au bout du compte, d'une aventure à ce point onéreuse qu'elle puisse en venir à compromettre la solidité financière du Mouvement. Toutes les précautions furent prises, évidemment, pour éviter ce scénario de catastrophe. Mais il y avait aussi l'insécurité du personnel, appelé à changer radicalement ses habitudes de travail et, par-dessus tout, menacé des réductions de personnel que semblait annoncer pareil projet, dans la foulée 
des grandes vagues d'informatisation et d'automatisation qui s'amorçaient, à l'époque, dans les usines.

31 À la faveur des besoins suscités par sa forte croissance, le Mouvement fit alors à ses employés la promesse formelle de ne faire aucune mise à pied pour des raisons liées à l'informatisation; au contraire, il s'engagea à former aux nouvelles tâches toutes les personnes qui accepteraient le défi et à entraîner à d'autres fonctions courantes dans le réseau celles qui se sentiraient incapables de s'adapter. Ces engagements dédramatisèrent la situation et, assurés du soutien et de la solidarité de l'organisation, les employés se mirent à l'apprentissage des nouveaux procédés.

On fut souvent surpris par la capacité d'adaptation du personnel. Nombre d'employés, y compris parmi les plus anciens, firent preuve d'une étonnante aptitude à ces fonctions nouvelles. Pour beaucoup, cette adaptation fut l'occasion d'une redécouverte d'euxmêmes et un prétexte pour montrer à leurs proches la versatilité de leurs talents. Tous ou presque, finalement, y prirent un goût nouveau pour les innovations techniques, puis une bonne dose de confiance et d'enthousiasme qui facilitera, pendant de nombreuses années, les changements qui se multipliaient.

De cette expérience majeure, le Mouvement gardera l'habitude de la consultation, de l'information, de la sensibilisation, de la foi en ses ressources et de la patience envers les cheminements lents. Ces enseignements seront souvent utilisés, par la suite, pour inciter les ressources humaines chargées de l'implantation d'autres changements à prendre le temps et les précautions nécessaires pour ne pas brusquer les démarches et laisser aux gens touchés par les changements la possibilité d'apprivoiser graduellement l'inconnu.

\section{Les difficultés du changement}

La boutade le dit bien, certaines personnes s'accommodent mieux de vieux problèmes que de solutions nouvelles. Il y a souvent, dans cette attitude, des traits de comportement liés au caractère ou au tempérament. Tous, cependant, ne refusent pas d'emblée le changement. Au contraire, en général, les êtres humains sont tendus vers le changement : tous souhaitent plus ou moins changer des choses, dans leur vie personnelle, leur milieu de travail ou leur entourage, de manière à améliorer leur bien-être, leur confort, leur niveau de satisfaction. Dans leur travail comme ailleurs, ils se montrent généralement intéressés à perfectionner les outils, les méthodes, les procédés de façon à rendre l'action plus facile, plus efficace, mieux adaptée aux besoins. Mais, pour s'engager dans un tel changement, il leur faut percevoir au moins sommairement les limites de la situation de départ, la possibilité de l'améliorer réellement et la pertinence des actions envisagées pour y parvenir.

Il demeure que la sécurité est un besoin fondamental de la personne et que, la vie obligeant à de multiples adaptations, nul n'est porté spontanément à changer une situation qu'il perçoit comme satisfaisante ou correcte. Bouddha avait compris ce trait de la psychologie humaine, lui qui prévenait affectueusement ses disciples qu'on ne pouvait refuser le constant renouvellement de la vie: «Rappelez-vous bien, mes enfants, leur disait-il, qu'il n'existe rien de constant si ce n'est le changement. »

Mais tous les changements ne sont pas aussi facilement acceptables, particulièrement ceux qui mettent en cause sa sécurité personnelle, les assises de son influence, de son 
pouvoir, de son avoir, ou encore celles des précieuses et trop rares certitudes qu'on est parvenu à se donner.

Si le Mouvement des caisses Desjardins a réussi à s'adapter à la plupart des changements auxquels le conviaient l'évolution du monde financier et celle de la société québécoise, il n'est pas encore parvenu à réaliser un autre changement qui semble s'imposer depuis une vingtaine d'années, celui de ses structures internes, et plus particulièrement la révision du partage des responsabilités entre les divers paliers de l'organisation.

$\mathrm{Au}$ fur et à mesure que l'expérience du Mouvement et que ses ressources financières, matérielles et techniques se sont accrues, les caisses, qui en sont les unités de base, ont augmenté leur capacité d'autonomie; en même temps, toutefois, la consolidation du réseau exigeait un resserrement de la solidarité et une uniformisation optimale des normes, services et procédés. Le même cheminement renforçait donc à la fois les capacités d'action de chacun des paliers et la nécessité de centraliser et d'uniformiser un certain nombre d'applications. Depuis une vingtaine d'années, on constate dans le réseau une certaine quantité de chevauchements et de doubles emplois que des objectifs de gestion rigoureuse devraient parvenir à éliminer ou tout au moins à réduire. D'un autre côté, la forte disparité dans la taille et les moyens actuels des fédérations entretient des différences considérables dans la qualité et la diversité des services que les caisses peuvent obtenir de leur organisme de regroupement régional; de plus, le processus démocratique se révèle souvent difficile d'application étant donné la disproportion des partenaires. Plusieurs études ont donc été faites sur ces sujets, plusieurs comités et commissions spéciales se sont penchés sur les problèmes, et la grande majorité des dirigeants et des employés s'entendent sur le diagnostic. C'est sur les solutions qu'on n'est jamais arrivé à faire un consensus suffisant pour permettre une action efficace et décisive.

Évidemment, dans une telle situation, chacun a la réaction normale de protéger son champ d'action et de juridiction. Chacun a le réflexe naturel de penser que c'est sa fonction à lui qui est nécessaire au bien de l'ensemble. Comme il reste toujours des choses à faire ou à améliorer, chaque dirigeant, chaque groupe de professionnels est à même de démontrer qu'il rend des services utiles, voire même indispensables. Les arguments font appel tantôt aux bénéfices de l'uniformisation, tantôt à ceux de la décentralisation, et tous ont leur part de crédibilité et de pertinence.

Les divers aspects du dossier sont maintenant suffisamment bien campés pour qu'on ne puisse plus esquiver longtemps la question, surtout que le resserrement des marges bénéficiaires forcera le Mouvement, comme les autres institutions financières, à une rigueur de gestion toujours plus grande. Les suites qui lui seront apportées seront significatives sur la capacité de l'institution de faire primer ses finalités sur les moyens progressivement mis en œuvre pour les réaliser et sur son aptitude à faire converger les légitimes aspirations individuelles et sectorielles vers l'intérêt commun. Il sera sans doute intéressant et instructif de voir comment se réaliseront les changements majeurs qu'appelle cette situation puisque, cette fois, ils risquent de toucher à la nature et à l'étendue du pouvoir et de l'influence d'un grand nombre d'acteurs. 


\section{Observations globales}

41 D'une observation attentive de l'évolution du Mouvement des caisses Desjardins au cours des vingt ou trente dernières années, on peut donc déduire notamment les enseignements suivants.

Premièrement, dans la gestion du changement, on a avantage à traiter les personnes comme des êtres intelligents, capables de comprendre les nécessités auxquelles fait face une entreprise ou une organisation, et capables d'adaptation et de responsabilité. Conséquemment, le changement ne doit pas être présenté comme une espèce de fatalité, mais comme un processus logique d'adaptation à un contexte ou à des conditions internes qui évoluent, et un processus que chaque partenaire a la capacité de comprendre et avantage à comprendre. La communication interne et les divers mécanismes de formation doivent s'attacher à expliquer sereinement les enjeux des changements à effectuer et les conséquences probables des divers scénarios possibles. Ce n'est pas nécessairement le changement que les gens refusent, mais le non-sens d'un changement qu'on ne leur a pas permis de comprendre et de considérer comme justifié, utile et souhaitable.

Deuxièmement, il y a souvent possibilité de faire surgir la volonté et la force de changement de la base même de l'organisation. Généralement, les artisans quotidiens de la production ou des services sont ceux et celles-là mêmes qui sont les mieux placés pour constater les lacunes de l'organisation du travail et celles des méthodes, procédés et processus utilisés. Quelques démarches simples de consultation suffisent, dans certains cas, à dégager des voies d'amélioration satisfaisantes. D'autres fois, il faudra compléter par des opérations de recherche plus complexes. Toujours, cependant, il y a lieu d'éviter que le discours interne ou externe ne présente les solutions envisagées comme des recettes miracles provenant de la tête de l'organisation ou de l'extérieur, c'est-à-dire, en d'autres mots, de sources relativement étrangères au contexte même où le changement devra être implanté. Une telle attitude est inévitablement perçue comme une expression de mépris, de méfiance ou de méconnaissance à l'égard de l'expérience et du savoir-faire des ressources humaines de l'organisation, et elle peut facilement devenir prétexte de conflit ou de résistance plus ou moins ouverte aux changements prévus. Même si la solution retenue n'est pas celle qui aurait suscité à l'intérieur l'adhésion la plus large, il est de prime importance que les gens se reconnaissent quelque part dans la solution préconisée. Il est généralement possible que le discours relatif aux changements à effectuer fasse au moins mention des préoccupations internes auxquelles la solution tente de donner suite, ou de certaines modalités d'application ou de mise en œuvre inspirées par des suggestions formulées par des gens de l'intérieur. D'ailleurs, plus le changement à réaliser est crucial pour l'organisation, plus on doit chercher à le faire accepter, désirer même, et plus on doit en multiplier les "porteurs" convaincus, y compris dans les catégories des « plus petits » ou plus humbles artisans.

Troisièmement, il est maladroit et contre-productif de dramatiser la nécessité du changement au point d'insécuriser inutilement les personnes qui auront à le réaliser. Certes, dans certaines situations, l'action s'impose et tout retard peut entraîner de lourdes conséquences. Il devient alors particulièrement indiqué de ménager les gens qui auront à assumer le défi en cause. Toute personne humaine a à faire face, dans sa vie quotidienne, à suffisamment de situations stressantes, sur divers plans, pour qu'il ne soit 
pas à propos d'ajouter délibérément aux pressions. Car si certaines personnes y réagissent bien, d'autres en perdent carrément leurs moyens, particulièrement si elles se sentent peu soutenues dans les cheminements à faire. Le changement peut souvent, au contraire, être présenté comme une occasion d'expériences nouvelles, d'apprentissages intéressants, de défis motivants, comme une aventure d'équipe où chacun aura sa part d'effort à fournir, mais aussi sa part de mérite.

Quatrièmement, en cette matière comme en bien d'autres, « le mieux est parfois l'ennemi du bien ». Les équipes d'experts nous ont habitués aux grandes planifications et aux plans directeurs globaux et à longue portée. Mais si les projets d'envergure peuvent stimuler, il leur arrive aussi de provoquer des craintes et des résistances. On a donc avantage à savoir dégonfler les défis posés par les grands plans par la prévision de plusieurs phases de réalisation. Les courtes haltes qui séparent ces étapes donnent aux gens le temps de reprendre leur souffle, et aux responsables la possibilité de réévaluer la pertinence des développements a venir et, au besoin, de corriger les perspectives.

Cinquièmement, il est toujours plus difficile, donc plus délicat, de faire accepter un recul qu'un bond en avant. Si une organisation a à gérer une décroissance ou à effectuer une rationalisation sévère de ses activités, le changement ne sera accepté que si toutes ses composantes ont manifestement à tenir compte des mêmes contraintes et à s'astreindre aux mêmes rigueurs.

Enfin, qu'on le reconnaisse ou pas, même les adultes les plus équilibrés ont un besoin naturel de la solidarité de leur entourage pour envisager des changements radicaux dans leur situation. Avec un peu de soutien, ils sont souvent capables de réalisations remarquables. Mais l'indifférence, la méfiance, le mépris ont tôt fait d'étouffer les meilleurs élans de créativité, de loyauté et d'engagement. Et si on peut se laisser gagner par l'attrait ou l'intérêt de causes ou de projets, on travaille aussi, inévitablement, pour les personnes qui ont la charge de les conduire; or, personne n'a le goût de se dépenser sans compter au profit de gens qui n'ont pour leurs collaborateurs aucune considération évidente et constante.

Les communications internes et externes d'une organisation sont toujours prégnantes de conceptions de la personne et de l'organisation qui ont une influence majeure sur la capacité et la motivation des personnes à entreprendre des changements à faire. Aucune stratégie ne saurait, à moyen ou à long terme, remplacer la solidarité réelle et profonde qui structure les équipes gagnantes ; car, en même temps qu'il sait canaliser les énergies au profit du renforcement d'une organisation, ce souci des personnes n'oublie jamais l'aspiration de chacune d'elles à sa part de sécurité, de succès, de croissance et de bonheur.

\section{BIBLIOGRAPHIE}

Beauchamp, M., La Communication et les organisations coopératives. Le cas du Mouvement des caisses Desjardins, Gaëtan Morin éditeur, Boucherville, 1989, 190 p. 
Giroux, N., Le Retournement stratégique : le cas de la carte Visa-Desjardins, thèse de doctorat, Montréal, Université du Québec à Montréal, 1990, 455 p.

Lévesque, G., Sheehy, G., Culture d'entreprise et informatisation chez Desjardins : un changement de paradigme, document de recherche, Université du Québec à Montréal, Chaire de coopération, $1990,28 \mathrm{p}$.

\section{RÉSUMÉS}

Comme institution de services financiers, le Mouvement des caisses Desjardins a été amené à réaliser, au cours des trente dernières années, un grand nombre de changements majeurs dans ses activités, ses services, ses méthodes et ses mécanismes de gestion et de contrôle. C'est dans ses pratiques coopératives, toutefois, et particulièrement à travers ses traditions de consultation et d'Information, qu'il a trouvé l'essentiel des dynamismes et de l'énergie pour mener à bien ces transformations et devenir un réseau financier solide, performant et profondément engagé dans l'évolution économique et sociale de la collectivité québécoise.

Over the last 30 years, the Mouvement des caisses Desjardins, as a financial services institution, has seen a need to operate a series of major adjustments to its activities, its services, its methods and its management and control procedures. Specifically by building on its cooperative practices. and particularly on its consultation and information traditions, the Mouvement found all the dynamism and energy that were needed to achieve such changes and to grow as a solid and highly performing financial network, one strongly committed to the economic and social development of Québec's people.

\section{AUTEUR}

\section{GUY CAMERON}

Diplômé en lettres et en pédagogie, l'auteur a enseigné pendant six ans. Il est par la suite entré, en 1977, au service de La Confédération des caisses populaires et d'économie Desjardins du Québec, où il a rempli successivement les fonctions d'agent d'information, de responsable des publications, de conseiller en communication, puis de conseiller en développement coopératif. 